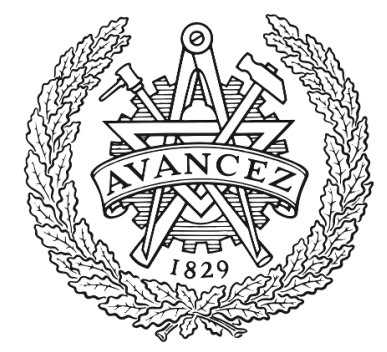

CHALMERS

UNIVERSITY OF TECHNOLOGY

\title{
Origin and evolution of surface spin current in topological insulators
}

Downloaded from: https://research.chalmers.se, 2023-04-26 11:29 UTC

Citation for the original published paper (version of record):

Dankert, A., Bhaskar, P., Khokhriakov, D. et al (2018). Origin and evolution of surface spin current in topological insulators. Physical Review B, 97(12). http://dx.doi.org/10.1103/PhysRevB.97.125414

N.B. When citing this work, cite the original published paper. 


\title{
Origin and evolution of surface spin current in topological insulators
}

\author{
André Dankert, ${ }^{1, *}$ Priyamvada Bhaskar, ${ }^{1}$ Dmitrii Khokhriakov, ${ }^{1}$ Isabel H. Rodrigues, ${ }^{1}$ Bogdan Karpiak, \\ M. Venkata Kamalakar, ${ }^{1,2}$ Sophie Charpentier, ${ }^{1}$ Ion Garate, ${ }^{3}$ and Saroj P. Dash ${ }^{1, \dagger}$ \\ ${ }^{1}$ Department of Microtechnology and Nanoscience, Chalmers University of Technology, \\ Quantum Device Laboratory, Göteborg 41296, Sweden \\ ${ }^{2}$ Department of Physics and Astronomy, Uppsala University, Box 516, SE-75120 Uppsala, Sweden \\ ${ }^{3}$ Département de Physique, Institut Quantique and Regroupement Québécois sur les Matériaux de Pointe, \\ Université de Sherbrooke, Sherbrooke, Québec, Canada J1K 2RI
}

(Received 24 November 2017; published 13 March 2018)

\begin{abstract}
The Dirac surface states of topological insulators offer a unique possibility for creating spin polarized charge currents due to the spin-momentum locking. Here we demonstrate that the control over the bulk and surface contribution is crucial to maximize the charge-to-spin conversion efficiency. We observe an enhancement of the spin signal due to surface-dominated spin polarization while freezing out the bulk conductivity in semiconducting $\mathrm{Bi}_{1.5} \mathrm{Sb}_{0.5} \mathrm{Te}_{1.7} \mathrm{Se}_{1.3}$ below $100 \mathrm{~K}$. Detailed measurements up to room temperature exhibit a strong reduction of the magnetoresistance signal between 2 and $100 \mathrm{~K}$, which we attribute to the thermal excitation of bulk carriers and to the electron-phonon coupling in the surface states. The presence and dominance of this effect up to room temperature is promising for spintronic science and technology.
\end{abstract}

DOI: 10.1103/PhysRevB.97.125414

Three-dimensional (3D) topological insulators (TIs) emerge as a result of band inversion due to strong spin-orbit (SO) coupling. These band inversions lead to the appearance of topologically protected gapless surface states, which have one spin state per momentum at the Fermi surface [Fig. 1(a)] [1-4]. The spins of carriers in TI surface states are locked perpendicular to their momenta [spin-momentum locking (SML)] enabling the creation of spin polarization by applying a charge current [Fig. 1(b)] [2,4]. These unique spin polarized surface states of TIs due to SML have been coupled to ferromagnetic contacts for creating giant spin transfer effects and for potentiometric detection of the current-induced spin polarization [5-12] even up to room temperature [13]. However, the interpretation of the results has remained challenging due to mixed contributions from the topological and trivial surface states on top of a bulk background conduction in prototype TIs such as $\mathrm{Bi}_{2} \mathrm{Se}_{3}, \mathrm{Bi}_{2} \mathrm{Te}_{3}$, and $\mathrm{Sb}_{2} \mathrm{Te}_{3}$, which have naturally occurring $\mathrm{Se}$ and $\mathrm{Te}$ vacancies resulting in unintentional doping [3]. These bulk carriers reduce the current-induced spin polarization in two ways: (i) by decreasing the fraction of the current flowing on the spin-momentum-locked surface states and (ii) by allowing the coupling between the topological surface states and the nontopological bulk and surface states. In particular, phonon-mediated surface-bulk coupling can lead to interband scattering between various spin-orbit states [dashed lines in Fig. 1(a) [14-17], which affects the detected surface spin polarization. These bulk-related problems have been improved upon by developing semiconducting bulk TIs $\left(\mathrm{Bi}_{x} \mathrm{Sb}_{1-x}\right)_{2} \mathrm{Te}_{3}$ and $\mathrm{Bi}_{1.5} \mathrm{Sb}_{0.5} \mathrm{Te}_{1.7} \mathrm{Se}_{1.3}$ (BSTS), where the surface state transport dominates at low temperature with

\footnotetext{
*andre.dankert@ chalmers.se

†saroj.dash@chalmers.se
}

parallel bulk conduction at high temperatures [18]. However, only a few studies reported the potentiometric detection of SML in such bulk semiconducting TIs, and were limited to cryogenic temperatures below $100 \mathrm{~K}[6,7]$. The electrical investigation of the SML phenomenon up to room temperature in such low doped TIs is crucial for the basic understanding of the influence of surface state and bulk band contribution to the detected magnetoresistance (MR) signal.

Here we study the surface spin polarization in BSTS with ferromagnetic tunnel contacts between 2 and $300 \mathrm{~K}$. The semiconducting bulk nature of BSTS thin films enables us to investigate SML in a surface-dominated transport regime at low temperatures and a surface-bulk coexistent (SBC) conduction regime at high temperatures. These results provide an understanding of the origin and evolution of SML signals with temperature as well as the contribution of bulk bands and topological surface states.

Device and electrical characterization of BSTS. The devices were prepared by depositing ferromagnetic (FM) tunnel contacts $\left(\mathrm{Co} / \mathrm{TiO}_{2}\right)$ on exfoliated BSTS flakes on a $\mathrm{SiO}_{2} / \mathrm{Si}$ substrate [Figs. 1(b) and 1(c), as well as Appendix 1) [13,19-21]. The tunneling characteristics of the contacts are presented in the Appendix, Fig. 6. The temperature dependence of BSTS channel resistance in a flake with a thickness $t=70 \mathrm{~nm}$ shows an increasing channel resistance from room temperature to $90 \mathrm{~K}$, and then decreasing below $90 \mathrm{~K}$ [Fig. 1(d)]. The increase in resistance when cooling down is expected for semiconducting BSTS flakes stemming from a parallel bulk contribution, whereas the reduction in resistance below $90 \mathrm{~K}$ indicates a freezing out of the charge carriers in the semiconducting bulk and increasingly dominating surface transport [22]. The freezing out of the bulk carrier states in our BSTS is further supported by Hall measurements showing a steady decrease of the charge carrier concentration with temperature (see Appendix, 
(a)

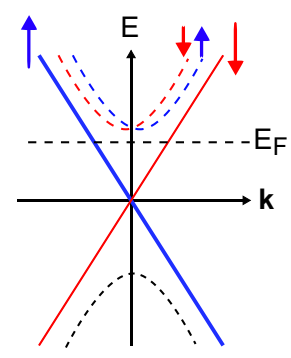

(c)

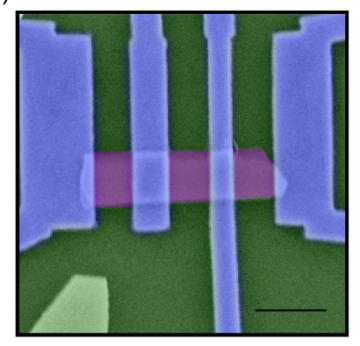

(b)

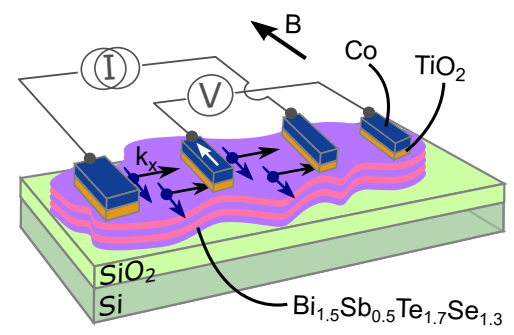

(d)

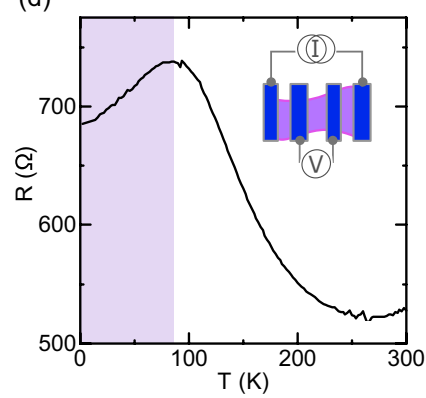

FIG. 1. Device schematic and electrical characterization. (a) Dirac cone of the topological surface state with spin-momentum locking (SML) and spin polarized nontopological surface states (dashed lines). (b) Schematic of a TI with FM tunnel contacts. The direction of spin current in $\mathrm{Bi}_{1.5} \mathrm{Sb}_{0.5} \mathrm{Te}_{1.7} \mathrm{Se}_{1.3}$ (BSTS) is defined by the charge current direction due to SML. (c) Colored scanning electron micrograph of a fabricated device with $\mathrm{Co} / \mathrm{TiO}_{2}$ contacts on an exfoliated BSTS flake (1 $\mu \mathrm{m}$ scale bar). (d) Temperature dependence of the BSTS channel resistance transitioning from metallic (colored background) to semiconducting (white background) behavior. Inset: measurement configuration.

Fig. 7). This indicates a remaining surface transport channel with higher mobility compared to the semiconducting bulk at low temperature. Using the measured charge carrier concentration and Fermi energy distribution, we can calculate the Fermi level to be within the band gap and about $22 \mathrm{meV}$ below the conduction band edge [23].

Magnetotransport and quantum oscillations. Magnetotransport measurements in BSTS show a weak antilocalization (WAL) behavior up to $55 \mathrm{~K}$ indicating a strong SO coupling (Appendix, Fig. 8). Using the Hikami-Larkin-Nagaoka (HLN) model, we can fit the conductivity correction $(\Delta \sigma)$ of the surface states with

$$
\Delta \sigma\left(B_{\perp}\right)=\alpha \frac{e^{2}}{\pi h}\left[\ln \frac{\hbar}{4 e l_{\varphi}^{2} B_{\perp}}-\psi\left(\frac{1}{2}+\frac{\hbar}{4 e l_{\varphi}^{2} B_{\perp}}\right)\right],
$$

where $\psi$ represents the digamma function, $l_{\varphi}$ is the phase coherence length, and $\alpha$ represents the dimensionality factor of the quantum system [24]. The temperature dependence of the phase coherence length $l_{\varphi} \propto T^{-0.61}$ above $10 \mathrm{~K}$ [Fig. 2(a)] indicates a $2 \mathrm{D}$ system with dominant phonon scattering $\left(l_{\varphi} \propto\right.$ $T^{-1 / 2}$ ), which is comparable to previous reports on surfacedominant BSTS [22] validating the usage of the HLN model. Figure 2(b) shows the measured conductivity at $2 \mathrm{~K}$ for an angle $\Theta$ from $0^{\circ}$ to $180^{\circ}$ plotted against the perpendicular magnetic field component $B_{\perp}=B \sin (\Theta)$. The angle inde-

(a)

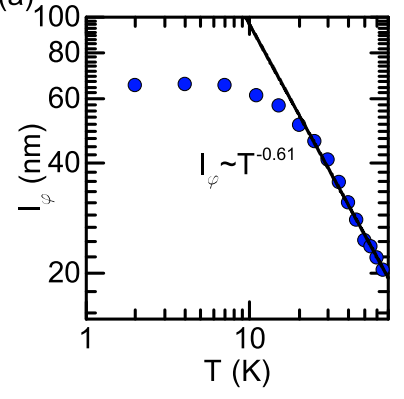

(c)

(b)

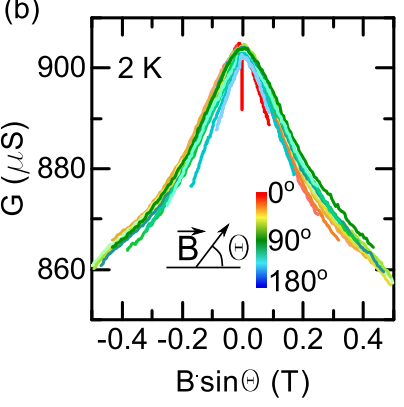

(d)

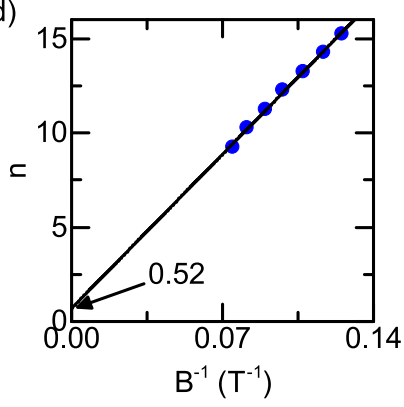

FIG. 2. Weak antilocalization (WAL) and Shubnikov-de Haas (SdH). (a) Temperature dependence of the phase coherence length. (b) Angle dependence of the WAL signal plotted against the out-ofplane magnetic field component at $2 \mathrm{~K}$. (c) Resistance dependence on $B^{-1}$ at $2 \mathrm{~K}$ measured at $I=1 \mu \mathrm{A}$ shows $\mathrm{SdH}$ oscillations. (d) The position of the oscillation's maxima extrapolates to $n(0)=\beta=0.52$.

pendence of this normalized WAL signal together with its short phase coherence length and its characteristic temperature dependence confirms the 2D surface states as the dominating transport channel at cryogenic temperatures.

In high magnetic field measurements, we also observed Shubnikov-de Haas ( $\mathrm{SdH}$ ) quantum oscillations in the longitudinal resistance with a perpendicular magnetic field up to $14 \mathrm{~T}$ at $2 \mathrm{~K}$. Figure 2(c) shows the oscillations as a function of the inverse magnetic field, where the parabolic magnetoresistive background has been subtracted. The respective fan diagram correlates the inverse magnetic fields at the maxima directly to different Landau levels $n=F / B+\beta$ [Fig. 2(d)], where slope $F$ is the oscillation frequency and offset $\beta$ is defined by the Berry phase $\varphi_{B}=2 \pi \beta$ [25]. We extract $\beta=0.52$ yielding $\varphi_{B} \approx \pi$, which is expected for the Dirac electrons in the topological surface states. Consequently, we can use the Onsager's relation $F=\frac{1}{2 \pi}\left(\frac{\hbar c}{2 \pi e}\right) \pi k_{F}^{2}=\left(\frac{\hbar c}{e}\right) n_{2 \mathrm{D}}$ to calculate the surface charge carrier concentration [4] $n_{2 \mathrm{D}}=5.9 \times 10^{12} \mathrm{~cm}^{-2}$, which is comparable to the charge carrier concentration extracted from Hall measurements (see Appendix 3). Since Hall measurements probe both bulk and surface transport, the identical charge carrier concentration from Hall and $\mathrm{SdH}$ measurements at $2 \mathrm{~K}$ implies a vanishing bulk background at low temperatures. The temperature and angle dependence of the WAL and the Berry phase of the $\mathrm{SdH}$ oscillations are clear evidence for a surface-dominated transport at low temperature.

Electrical detection of SML. BSTS with a dominant surface transport is ideal to study the contribution to the magnetoresistance signal due to SML. Figure 3(a) shows the measurement principle on a multiterminal BSTS device. By applying a 
(a)

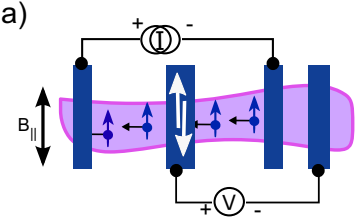

(c)

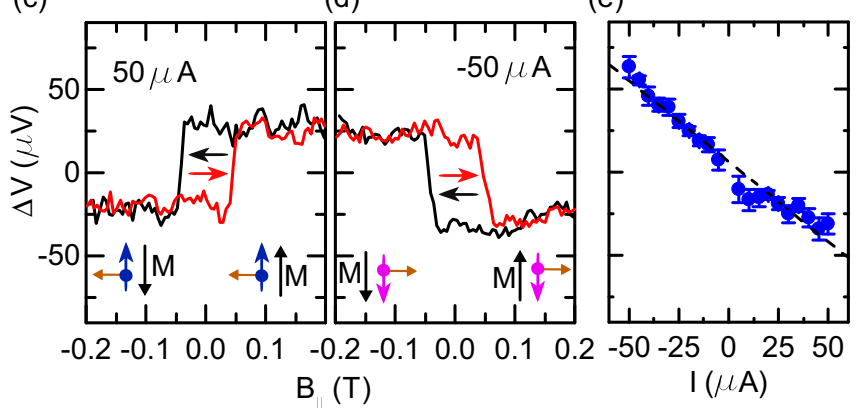

FIG. 3. Electrical detection of SML. Schematics of the magnetoresistance measurements between current-induced BSTS surface spins, $S_{\uparrow}$ in (a) and $S_{\downarrow}$ in (b), and magnetization $(\vec{M})$ of the detector FM contact [13]. (c) Spin signal shows a hysteretic switching at $2 \mathrm{~K}$ for a bias current of $+50 \mu \mathrm{A}$. The arrows show the magnetic field sweep directions up (red) and down (black). (d) Reversing the current direction $\left(-50 \mu \mathrm{A}\right.$ locking to $\left.S_{\downarrow}\right)$ results in a spin signal with an inverted hysteretic switching. (e) Bias current dependence of the spin signal amplitude $\Delta V=\left(\mu_{\uparrow}-\mu_{\downarrow}\right) /-e$ measured at $10 \mathrm{~K}$ for BSTS.

charge current we generate a spin polarization $\left(P_{S}\right)$ in the BSTS surface states due to SML. This net spin polarization beneath the FM tunnel contact $\left(\mathrm{Co} / \mathrm{TiO}_{2}\right)$ is detected as a voltage signal depending on parallel or antiparallel alignment of the FM and the spin orientation. Sweeping the in-plane magnetic field, the magnetization of the FM detector is switched with respect to $P_{S}$ yielding a step in the voltage signal. An up and down sweep of the magnetic field results in a hysteretic switching presented in Fig. 3(c), as measured on a BSTS flake of $70 \mathrm{~nm}$ thickness at $2 \mathrm{~K}$ by applying a dc current of $+50 \mu \mathrm{A}$. Similarly, the spin polarization in the BSTS surface states can be flipped by inverting the current direction $[-50 \mu \mathrm{A}$ in Fig. 3(b)], resulting in a reversed spin signal [Fig. 3(d)] [13]. Measuring the full bias range, we observe a linear dependence of the spin signal $\Delta V$ [Fig. 3(e)], as expected, since the spin density scales linearly with the current density [14]. However, the strong spin-orbit coupling in the TI as well as stray fields at the magnetic contacts can yield contributions from Rashba states [3], spin Hall or stray Hall effects [10,26], mimicking a SML signal. Previously, such spurious effects have been ruled out by several control experiments using angle [13], gate [8], and carrier dependent $[11,27]$ measurements. In particular the stray Hall effect is strongly charge carrier type dependent, whereas the spin locking is not. Therefore, we studied $\mathrm{Sb}_{2} \mathrm{Te}_{3}$, a known $p$-type TI, to confirm the sign of the detected MR signal (see Appendix 6). The observed SML signal matches our data reported here on BSTS, as well as previous studies on $\mathrm{Bi}_{2} \mathrm{Se}_{3}$ and BST [6-8,13], which confirms the signal origin stemming from the SML in the TI surface states. Additionally, we observe an enhanced spin resistance $R_{S}=\Delta V / I$ up to $1.5 \Omega$ in BSTS at low temperature, which is orders of magnitude higher than aforementioned spin Hall and stray Hall effects

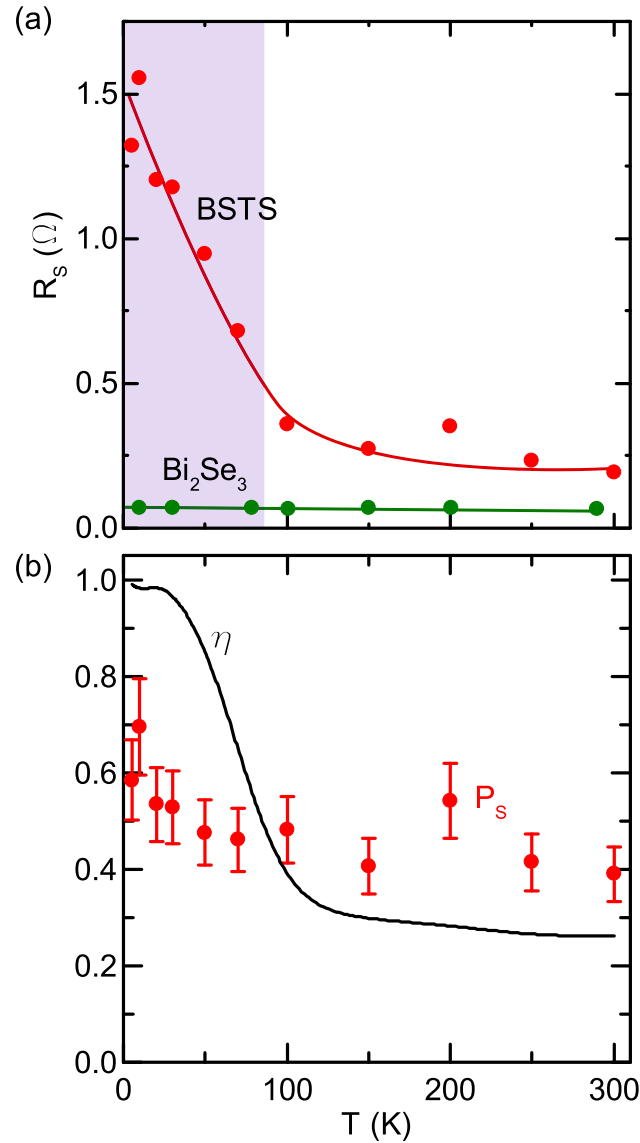

FIG. 4. Temperature dependence of SML signal. (a) Temperature dependence of the spin signal amplitude $R_{S}=\Delta V / I$ for BSTS compared with $\mathrm{Bi}_{2} \mathrm{Se}_{3}$ [13] and correlated with the channel's metalsemiconductor transition (colored background: SML; white background: SBC). The solid lines are guides to the eye. (b) Temperature dependence of the surface contribution factor $\eta=G^{S} /\left(G^{S}+G^{B}\right)$ and the surface spin polarization $P_{S}$.

[8]. Furthermore, such a high $R_{S}$ is also at least one order of magnitude higher than previously reported SML results on metallically doped TIs [5,13], comparable to reports on BSTS [6], and can be attributed to the reduced number of bulk carriers at low temperature.

In order to understand the origin of the enhancement of the signal at low temperature, we carried out a detailed temperature dependence measurement of the magnetoresistance. We observed a clear MR switching up to room temperature, which decreases drastically when warming up from 2 to $100 \mathrm{~K}$ and levels off in the SBC regime 100-300 K [Fig. 4(a) and Appendix, Fig. 9]. Previous reports on potentiometric measurements on BSTS show a similar temperature dependence $[6,18]$, however, in a low temperature range. In contrast, on highly doped TIs, different studies [5,7,8,13,28,29] showed only a weak temperature dependence over large temperature ranges (see also Appendix, Fig. 10).

In our case, the change in the temperature dependence at around $100 \mathrm{~K}$ coincides with the transition from surface to SBC transport behavior [Fig. 1(d)]. Below the activation gap, the number of bulk carriers and the inelastic scattering rate of surface states increase exponentially with temperature $[8,30]$. 
This temperature dependence becomes polynomial when the temperature exceeds the activation gap, as seen in Fig. 4(a) $[13,31]$. This indicates that the detected signal stems from SML in the surface states partly suppressed by a temperature dependent surface-bulk scattering and thermally activated bulk charge carriers $[10,14,16]$.

Next, we fit the temperature-dependent resistance shown in Fig. 1(d) to a two-channel transport model. In this model, the thermally activated bulk conductance is given by $G^{B}(T)=t\left(\rho_{B} \exp (\Delta / k T)\right)^{-1}$, where $k$ is the Boltzmann constant, $\rho_{B}$ is the high-temperature bulk resistivity, and $\Delta$ is the activation gap between the Fermi level and the closest bulk states. Similarly, the surface state conductance reads

$$
G^{S}(T)= \begin{cases}\left(R^{*}+B T^{4}+C T^{5}\right)^{-1}, & T<T_{\mathrm{BG}}, \\ \left(R^{*}+A T\right)^{-1}, & T>T_{\mathrm{BG}} .\end{cases}
$$

The surface resistance $R^{*}=R_{0}+D \exp (\Delta / k T)$, with the low temperature residual resistance $R_{0}$ and an exponential increase with temperature of the scattering from surface to bulk states $(D)$, is dominated by nonlinear phonon contribution parameters $(B$ and $C$ ) at low temperatures (below the BlochGrüneisen temperature $T_{\mathrm{BG}}=2 \hbar v_{s} k_{\mathrm{F}} / k_{\mathrm{B}} \approx 10 \mathrm{~K}$ ) [32] and the linear electron-phonon scattering parameter $A$ for $T>$ $T_{\mathrm{BG}}$. From the fitting, one may extract the fraction of the current that flows on the surface states, $\eta=G^{S} /\left(G^{S}+G^{B}\right)$. This fraction decreases rapidly from $100 \%$ at low temperature to about $30 \%$ at $100 \mathrm{~K}$, beyond which it remains almost constant up to $300 \mathrm{~K}$ [Fig. 4(b)]. In contrast to previous transport models [33], this behavior matches our experimentally observed resistance change and theoretical expectations of a thermally activated dominant bulk conduction in thin-film TIs well.

Finally, we can estimate the spin resistance $R_{S}$, due to SML induced by a current $I_{S}=\eta I$ yielding the surface spin polarization $P_{S}$, which can be expressed as

$$
R_{s}=\frac{\Delta V}{I}=\eta R_{B T} P_{S} P_{\mathrm{FM}}
$$

where $P_{\mathrm{FM}}$ is the polarization of the FM detector and $R_{B T}$ is the ballistic resistance [14]. The former has been found in previous studies to have an upper limit of $P_{\mathrm{FM}}=20 \%$ for our $\mathrm{Co} / \mathrm{TiO}_{2}$ contacts $[13,34]$. The ballistic conductance $\frac{1}{R_{B T}}$ equals the quantum of conductance $\frac{q^{2}}{h}$ multiplied by the number of propagating modes $\frac{k_{\mathrm{F}} W}{\pi}$, where $W$ is the width of the conductance channel. The Fermi wave number $k_{\mathrm{F}}$ can be derived from the 2D charge carrier concentration $n_{2 \mathrm{D}}$ as $k_{\mathrm{F}}=\sqrt{4 \pi n_{2 \mathrm{D}}}$ [13]. Using Eq. (3) and the surface charge carrier concentrations measured in $\mathrm{SdH}$ and Hall measurements, we calculate a current-induced surface spin polarization $P_{S} \approx 0.4-0.6$, which is almost constant within the error margins over the full temperature range [Fig. 4(b)] and twice as high as previously reported $[5,13,16,35]$. The deviation from the optimal $P_{S}=1$ originates from spin-orbit entanglement in the topological surface states [16], as well as the possible occurrence of nontopological surface states at the Fermi level $[36,37]$. Optimizing the crystal growth and doping could yield a lower bulk conduction and hence a high surface spin signal under ambient conditions, which would present a serious competition for ferromagnetic contacts in spintronic applications.

In summary, we have presented the electrical detection of spin polarized surface currents due to SML on BSTS by FM tunnel contacts over a broad temperature range up to $300 \mathrm{~K}$. High quality semiconducting BSTS crystals and FM tunnel contacts allowed the observation of a spin signal of up to $1.5 \Omega$, which is at least one order of magnitude higher than previously reported results on metallically doped TIs in the same temperature range [13,27]. The large magnitude of the signal and its sign, combined with quantum transport measurements, prove clearly the SML originating from surface states at low temperature. Furthermore, we observe a strong temperature dependence of the SML signal up to $100 \mathrm{~K}$, thereafter remaining constant up to room temperature. A two-channel transport model considering thermally activated bulk carriers and surface-to-bulk scattering confirms an almost temperature independent surface spin polarization $P_{S}$. This elucidates the influence of the bulk conduction and scattering mechanisms that suppress the detected SML signal, whilst still observable up to room temperature [13].

\section{ACKNOWLEDGMENTS}

The authors acknowledge financial support from the European Union's Horizon 2020 research and innovation programme under Grant No. 696656 (Graphene Flagship), EU FlagEra project (from Swedish Research council VR No. 2015-06813), Swedish Research Council VR project grants (No. 2016-03658), and Graphene center and the AoA Nano program at Chalmers University of Technology. The authors also acknowledge the support of colleagues at the Quantum Device Physics Laboratory and Nanofabrication Laboratory at Chalmers University of Technology. I.G. acknowledges financial support from the Canada First Research Excellence Fund and the Natural Sciences and Engineering Research Council of Canada.

\section{APPENDIX}

\section{Fabrication and characterization}

The $\mathrm{Bi}_{1.5} \mathrm{Sb}_{0.5} \mathrm{Te}_{1.7} \mathrm{Se}_{1.3}$ flakes were exfoliated from a bulk crystal, using the conventional Scotch tape cleavage technique, onto a clean $\mathrm{SiO}_{2}(285 \mathrm{~nm}) /$ highly doped $n$-type $\mathrm{Si}$ substrate. The crystal was obtained from Miracrys, grown from a melt using a high vertical Bridgeman method [13]. The flakes were identified using optical microscopy and the flake thickness, uniformity, and material quality was analyzed with atomic force microscopy and Raman spectroscopy, respectively [Figs. 5(a) and 5(b)]. This revealed homogeneous $70 \mathrm{~nm}$ thick flakes with widths of about $1 \mu \mathrm{m}$ and a characteristic Raman spectrum for BSTS [38]. Electrodes were patterned by electron beam lithography. The contact deposition was performed in an ultrahigh vacuum electron beam evaporator after an in situ surface cleaning using low power argon ion plasma for $10 \mathrm{~s}$. Electrodes with widths $0.3-1 \mu \mathrm{m}$ and channel length of $0.2-1 \mu \mathrm{m}$ are used. As contact material, we used $\mathrm{TiO}_{2} / \mathrm{Co}$ for the detection of spin-momentum locking. The $\approx 1.5 \mathrm{~nm} \mathrm{TiO}_{2}$ 
(a)

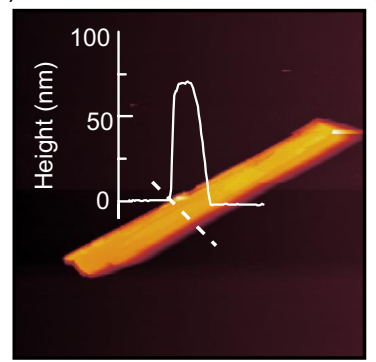

(b)

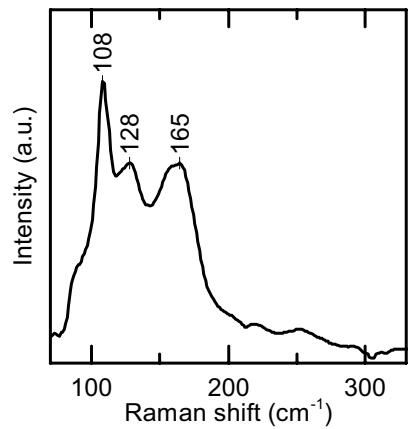

FIG. 5. Characterization of BSTS. (a) Atomic force micrograph of a $70 \mathrm{~nm}$ thick BSTS flake. Inset: height profile along the dashed line. (b) Raman spectrum of a $70 \mathrm{~nm}$ BSTS flake.

tunnel barrier was deposited by electron beam evaporation and in situ oxidation using a pure oxygen atmosphere. The BSTS devices were measured with a Quantum Design Physical Property Measurement System (PPMS) with resistivity option using direct current (dc).

\section{Characterization of ferromagnetic tunnel junctions}

The tunneling properties of the ferromagnetic (FM) tunnel contacts are characterized in a three-terminal measurement configuration (Fig. 6). The current-voltage characteristics exhibit a nonlinear behavior typical for tunneling transport. Furthermore, the temperature dependence shows an increase of the resistance by only a factor of 5 with decrease in temperature indicating a good quality $\mathrm{TiO}_{2}$ tunnel barrier on the BSTS flakes.

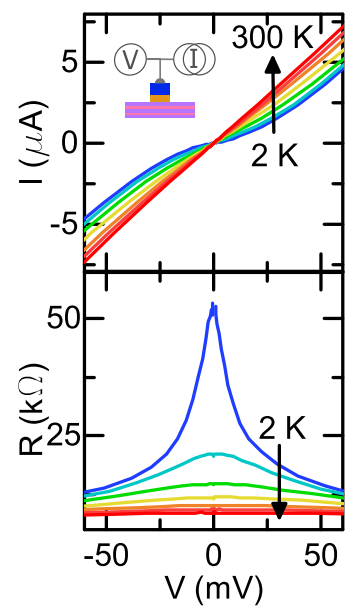

FIG. 6. Characterization of ferromagnetic tunnel junctions. Temperature dependence of the contact current-voltage characteristic (top panel) and resistance curves (bottom panel). The nonlinearity indicates a good tunnel barrier with a weak temperature dependence.

(a)

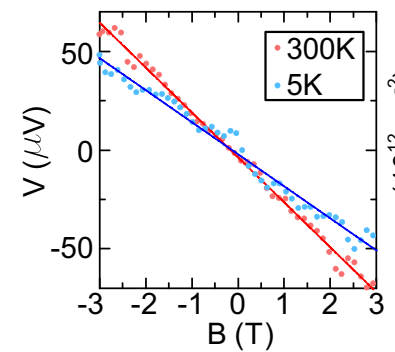

(b)

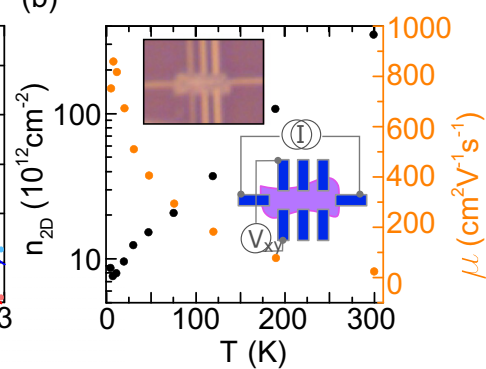

FIG. 7. Hall measurement in BSTS. (a) The Hall voltage measured in BSTS using a bias current of $10 \mu \mathrm{A}$ at room temperature and $5 \mathrm{~K}$. (b) Temperature dependence of the Hall charge carrier concentration $n$ and mobility $\mu$. Insets: optical microscope image of Hall device and measurement schematic.

\section{Temperature dependence of carrier mobility and concentration}

Hall measurements were performed on a $\mathrm{Bi}_{1.5} \mathrm{Sb}_{0.5} \mathrm{Te}_{1.7} \mathrm{Se}_{1.3}$ channel between $2 \mathrm{~K}$ and $300 \mathrm{~K}$ (Fig. 7). By applying a perpendicular magnetic field the Hall voltage across the channel width is detected, allowing us to extract the 2D charge carrier concentration $n_{2 \mathrm{D}}$ and mobility $\mu$. Figure 7 shows a low $n_{2 \mathrm{D}}$ at $2 \mathrm{~K}$, comparable to the surface charge carrier concentration extracted from Shubnikov-de Haas measurements (see main manuscript), and increasing with temperature, due to thermally excited carriers from the bulk. The mobility at room temperature is about $25 \mathrm{~cm}^{2}(\mathrm{~V} \mathrm{~s})^{-1}$, and increases to $825 \mathrm{~cm}^{2}(\mathrm{~V} \mathrm{~s})^{-1}$ at $2 \mathrm{~K}$. This behavior matches well with an intrinsically low doped semiconductor freeze-out in the bulk at low temperatures with a remaining charge carrier concentration stemming from the surface states at $2 \mathrm{~K}$.

\section{Weak antilocalization}

The quantum transport properties of BSTS were measured by sweeping an out-of-plane magnetic field while measuring the lateral channel resistance. We observe a correction of the magnetoresistance as expected for strong SO coupled materials resulting in weak antilocalization up to $55 \mathrm{~K}$ (Fig. 8).

\section{Temperature dependence of SML signals}

We carried out detailed temperature dependence measurements of the SML signal and observed a clear switching up to room temperature, which decreases drastically when warming up from 2 to $100 \mathrm{~K}$ and levels off in the SBC regime 100-300 K (Fig. 9).

\section{Comparison of SML signals}

The amplitude and the temperature dependence of the SML signal in our BSTS devices are compared in Fig. 10 with literature values since the first report of electrical SML detection by $\mathrm{Li}$ et al. [5], and various studies on $\mathrm{Bi}_{2} \mathrm{Se}_{3}$ (Liu et al. [8], Dankert et al. [13], and Li et al. [27]), in $\left(\mathrm{Bi}_{0.53} \mathrm{Sb}_{0.47}\right)_{2} \mathrm{Te}_{3}$ (Tang et al. [7]) and $\mathrm{Bi}_{1.5} \mathrm{Sb}_{0.5} \mathrm{Te}_{1.7} \mathrm{Se}_{1.3}$ (this paper and Ando et al. [6]). The SML signal voltage is compared as spin resistance. Our SML signal amplitude 


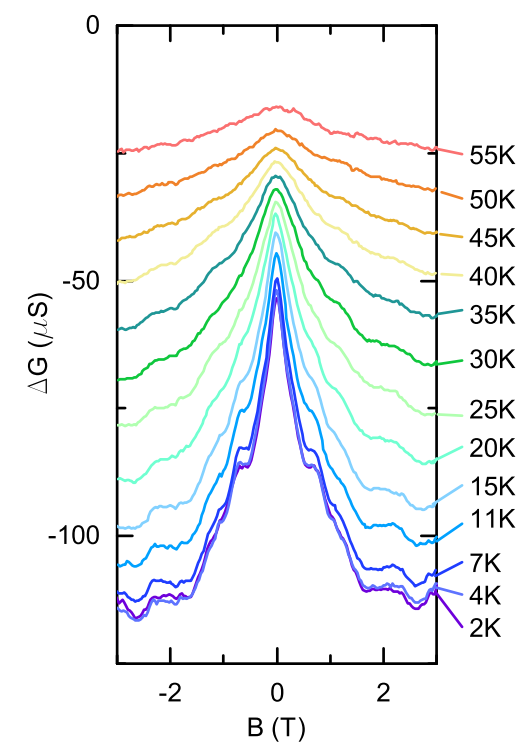

FIG. 8. Weak antilocalization (WAL). Magnetoconductance measurements of BSTS with applied perpendicular magnetic field showing WAL up to $55 \mathrm{~K}$ measured at $I=5 \mu \mathrm{A}$. A background without WAL signal measured at $70 \mathrm{~K}$ has been subtracted.

is at least one order of magnitude higher than reported in metallically doped $\mathrm{Bi}_{2} \mathrm{Se}_{3}$. The temperature dependence of our BSTS sample spans the full temperature range of $2-300 \mathrm{~K}$. Only two other studies on metallic $\mathrm{Bi}_{2} \mathrm{Se}_{3}$ cover a similar range $[13,27]$. Below $100 \mathrm{~K}$, the strong signal increase in our measurement matches well the results on semiconducting BSTS [6] and BST [7]. All studies on metallic $\mathrm{Bi}_{2} \mathrm{Se}_{3}$ show a weak temperature dependence, whereas the variation presented in studies with low detected spin potential splitting [5,27] may be due to other influences and the temperature dependence is also negligible compared to BSTS.

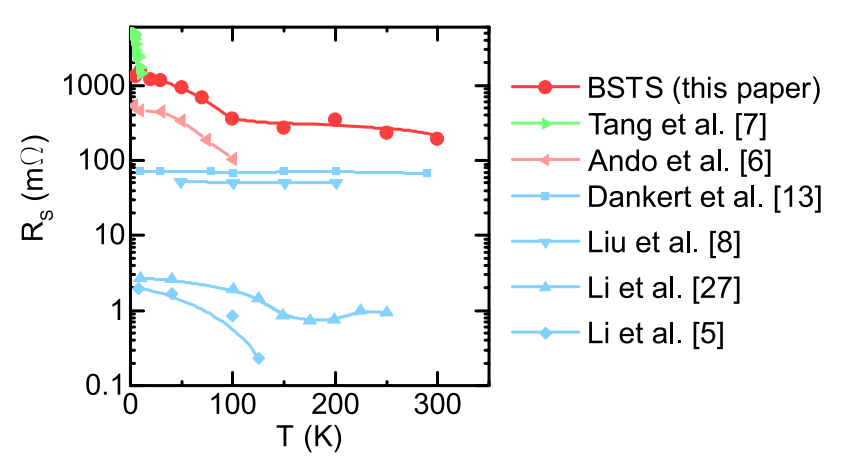

FIG. 10. Comparison of various data sets of SML measurements from literature. The temperature dependences of the spin resistance observed in several TI compounds published since the first report of electrical SML detection by Li et al. [5], various reports on $\mathrm{Bi}_{2} \mathrm{Se}_{3}$ (Liu et al. [8], Dankert et al. [13], and Li et al. [27]), in $\left(\mathrm{Bi}_{0.53} \mathrm{Sb}_{0.47}\right)_{2} \mathrm{Te}_{3}$ (Tang et al. [7]) and $\mathrm{Bi}_{1.5} \mathrm{Sb}_{0.5} \mathrm{Te}_{1.7} \mathrm{Se}_{1.3}$ (this paper and Ando et al. [6]).

\section{Spin-momentum locking in $p$-type $\mathrm{TI} \mathrm{Sb}_{2} \mathrm{Te}_{3}$}

The stray fields at the magnetic contacts can yield stray Hall effects, which can appear similar to the SML signal. However, such spurious signals are strongly charge carrier dependent and change sign when switching from electron to hole transport. In contrast, the spin polarization of the SML does not depend on the charge carriers, since the locking as well as the momentum are opposite for holes and electrons. We studied $\mathrm{Sb}_{2} \mathrm{Te}_{3}$, a known $p$-type $\mathrm{TI}$, to verify the origin of the measured signal. Figure 11(a) shows the Hall measurement in $\mathrm{Sb}_{2} \mathrm{Te}_{3}$, which exhibits a majority hole carrier concentration of $p=1.16 \times 10^{14} \mathrm{~cm}^{-2}$. The observed SML signal for positive [Fig. 11(b)] and negative [Fig. 11(c)] bias current shows the typical hysteretic switching and matches our data reported here on BSTS, as well as previous studies on $\mathrm{Bi}_{2} \mathrm{Se}_{3}$ and $\mathrm{BST}$ $[6-8,13]$, which confirms the signal origin stemming from the SML in the TI surface states.

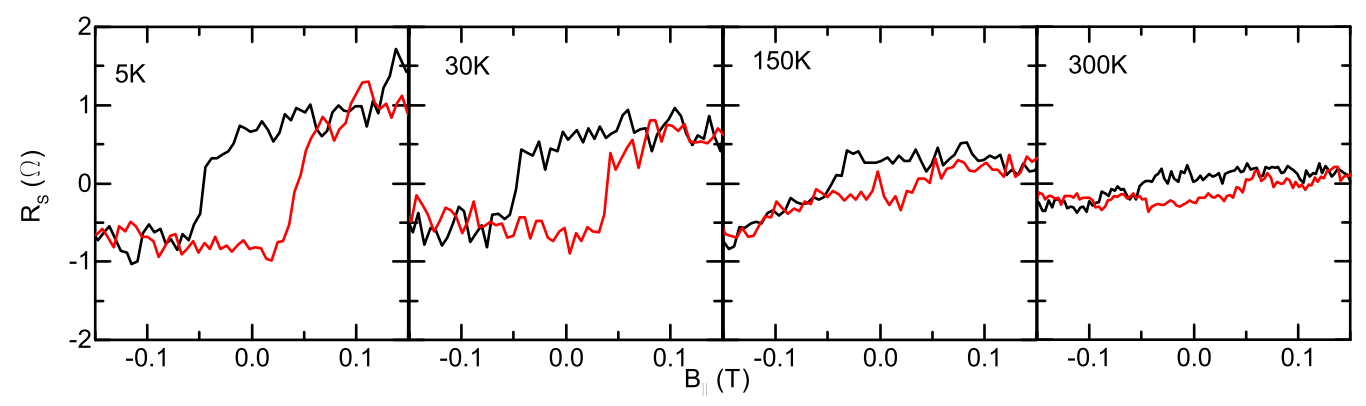

FIG. 9. Temperature dependence of SML signal. Spin signal measured at different temperatures at $20 \mu \mathrm{A}$ for BSTS. 
(a)

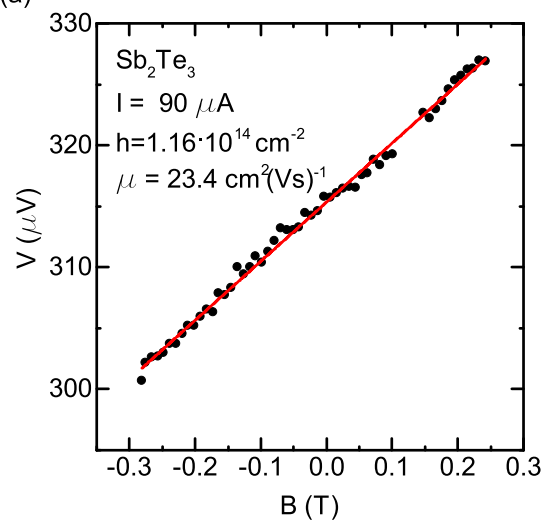

(b)

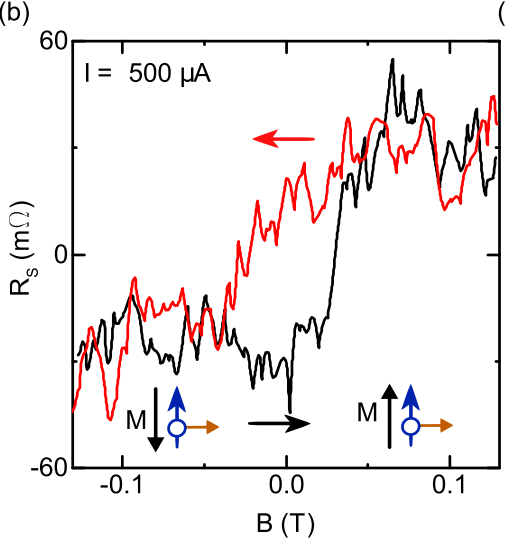

(c)

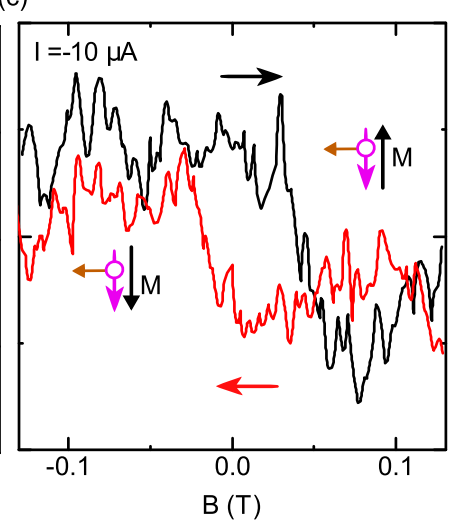

FIG. 11. Spin-momentum locking in $p$-type $\mathrm{Sb}_{2} \mathrm{Te}_{3}$. (a) Hall measurement showing $p$-type doping. SML measured at opposite bias polarities of (b) $500 \mu \mathrm{A}$ and (c) $-10 \mu \mathrm{A}$.

[1] Y. Xia, D. Qian, D. Hsieh, L. Wray, A. Pal, H. Lin, A. Bansil, D. Grauer, Y. S. Hor, R. J. Cava, and M. Z. Hasan, Nat. Phys. 5, 398 (2009).

[2] M. Z. Hasan and C. L. Kane, Rev. Mod. Phys. 82, 3045 (2010).

[3] D. Pesin and A. H. MacDonald, Nat. Mater. 11, 409 (2012).

[4] Y. Ando, J. Phys. Soc. Jpn. 82, 102001 (2013).

[5] C. H. Li, O. M. J. van 't Erve, J. T. Robinson, Y. Liu, L. Li, and B. T. Jonker, Nat. Nanotechnol. 9, 218 (2014).

[6] Y. Ando, T. Hamasaki, T. Kurokawa, K. Ichiba, F. Yang, M. Novak, S. Sasaki, K. Segawa, Y. Ando, and M. Shiraishi, Nano Lett. 14, 6226 (2014).

[7] J. Tang, L.-T. Chang, X. Kou, K. Murata, E. S. Choi, M. Lang, Y. Fan, Y. Jiang, M. Montazeri, W. Jiang, Y. Wang, L. He, and K. L. Wang, Nano Lett. 14, 5423 (2014).

[8] L. Liu, A. Richardella, I. Garate, Y. Zhu, N. Samarth, and C.-T. Chen, Phys. Rev. B 91, 235437 (2015).

[9] J. Tian, I. Miotkowski, S. Hong, and Y. P. Chen, Sci. Rep. 5, 14293 (2015).

[10] E. K. de Vries, A. M. Kamerbeek, N. Koirala, M. Brahlek, M. Salehi, S. Oh, B. J. van Wees, and T. Banerjee, Phys. Rev. B 92, 201102 (2015).

[11] C. H. Li, O. M. J. van 't Erve, Y. Y. Li, L. Li, and B. T. Jonker, Sci. Rep. 6, 29533 (2016).

[12] F. Yang, S. Ghatak, A. A. Taskin, K. Segawa, Y. Ando, M. Shiraishi, Y. Kanai, K. Matsumoto, A. Rosch, and Y. Ando, Phys. Rev. B 94, 075304 (2016).

[13] A. Dankert, J. Geurs, M. V. Kamalakar, S. Charpentier, and S. P. Dash, Nano Lett. 15, 7976 (2015).

[14] S. Hong, V. Diep, S. Datta, and Y. P. Chen, Phys. Rev. B 86, 085131 (2012).

[15] P. R. Hammar and M. Johnson, Phys. Rev. B 61, 7207 (2000).

[16] O. V. Yazyev, J. E. Moore, and S. G. Louie, Phys. Rev. Lett. 105, 266806 (2010).

[17] C. Datzer, A. Zumbülte, J. Braun, T. Förster, A. B. Schmidt, J. Mi, B. Iversen, P. Hofmann, J. Minár, H. Ebert, P. Krüger, M. Rohlfing, and M. Donath, Phys. Rev. B 95, 115401 (2017).
[18] Z. Ren, A. A. Taskin, S. Sasaki, K. Segawa, and Y. Ando, Phys. Rev. B 84, 165311 (2011).

[19] A. Dankert, L. Langouche, M. V. Kamalakar, and S. P. Dash, ACS Nano 8, 476 (2014).

[20] A. Fert, Rev. Mod. Phys. 80, 1517 (2008).

[21] S. P. Dash, S. Sharma, R. S. Patel, M. P. de Jong, and R. Jansen, Nature (London) 462, 491 (2009).

[22] B. Xia, P. Ren, A. Sulaev, P. Liu, S.-Q. Shen, and L. Wang, Phys. Rev. B 87, 085442 (2013).

[23] K. Kondou, R. Yoshimi, A. Tsukazaki, Y. Fukuma, J. Matsuno, K. S. Takahashi, M. Kawasaki, Y. Tokura, and Y. Otani, Nat. Phys. 12, 1027 (2016).

[24] S. Hikami, A. I. Larkin, and Y. Nagaoka, Prog. Theor. Phys. 63, 707 (1980).

[25] K. S. Novoselov, A. K. Geim, S. V. Morozov, D. Jiang, M. I. Katsnelson, I. V. Grigorieva, S. V. Dubonos, and A. A. Firsov, Nature (London) 438, 197 (2005).

[26] I. Appelbaum, H. N. Tinkey, and P. Li, Phys. Rev. B 90, 220402(R) (2014).

[27] C. H. Li, O. M. J. van 't Erve, S. Rajput, L. Li, and B. T. Jonker, Nat. Commun. 7, 13518 (2016).

[28] A. R. Mellnik, J. S. Lee, A. Richardella, J. L. Grab, P. J. Mintun, M. H. Fischer, A. Vaezi, A. Manchon, E.-A. Kim, N. Samarth, and D. C. Ralph, Nature (London) 511, 449 (2014).

[29] M. Jamali, J. S. Lee, J. S. Jeong, F. Mahfouzi, Y. Lv, Z. Zhao, B. K. Nikolić, K. A. Mkhoyan, N. Samarth, and J.-P. Wang, Nano Lett. 15, 7126 (2015).

[30] K. Saha and I. Garate, Phys. Rev. B 90, 245418 (2014).

[31] A. Dankert, R. S. Dulal, and S. P. Dash, Sci. Rep. 3, 3196 (2013).

[32] S. Giraud, A. Kundu, and R. Egger, Phys. Rev. B 85, 035441 (2012).

[33] Y. Xu, I. Miotkowski, C. Liu, J. Tian, H. Nam, N. Alidoust, J. Hu, C.-K. Shih, M. Z. Hasan, and Y. P. Chen, Nat. Phys. 10, 956 (2014).

[34] M. V. Kamalakar, C. Groenveld, A. Dankert, and S. P. Dash, Nat. Commun. 6, 6766 (2015).

[35] D. Hsieh, Y. Xia, D. Qian, L. Wray, J. H. Dil, F. Meier, J. Osterwalder, L. Patthey, J. G. Checkelsky, N. P. Ong, A. V. 
Fedorov, H. Lin, A. Bansil, D. Grauer, Y. S. Hor, R. J. Cava, and M. Z. Hasan, Nature (London) 460, 1101 (2009).

[36] M. S. Bahramy, P. D. C. King, A. de la Torre, J. Chang, M. Shi, L. Patthey, G. Balakrishnan, P. Hofmann, R. Arita, N. Nagaosa, and F. Baumberger, Nat. Commun. 3, 1159 (2012).
[37] M. Bianchi, D. Guan, S. Bao, J. Mi, B. B. Iversen, P. D. C. King, and P. Hofmann, Nat. Commun. 1, 128 (2010).

[38] N. H. Tu, Y. Tanabe, K. K. Huynh, Y. Sato, H. Oguro, S. Heguri, K. Tsuda, M. Terauchi, K. Watanabe, and K. Tanigaki, Appl. Phys. Lett. 105, 063104 (2014). 